\title{
Multi-institutional comparison of volumetric modulated arc therapy vs. intensity-modulated radiation therapy for head-and-neck cancer: a planning study
}

\author{
Andrea Holt ${ }^{1,7}$, Dirk Van Gestel ${ }^{2}$, Mark P Arends ${ }^{3}$, Erik W Korevaarr ${ }^{4}$, Danny Schuring ${ }^{5}$, Martina C Kunze-Busch ${ }^{6}$,
} Rob JW Louwe ${ }^{6,8}$ and Corine van Vliet-Vroegindeweij ${ }^{*}$

\begin{abstract}
Background: Compared to static beam Intensity-Modulated Radiation Therapy (IMRT), the main advantage of Volumetric Modulated Arc Therapy (VMAT) is a shortened delivery time, which leads to improved patient comfort and possibly smaller intra-fraction movements. This study aims at a treatment planner-independent comparison of radiotherapy treatment planning of IMRT and VMAT for head-and-neck cancer performed by several institutes and based on the same CT- and contouring data.

Methods: Five institutes generated IMRT and VMAT plans for five oropharyngeal cancer patients using either Pinnacle ${ }^{3}$ or Oncentra Masterplan to be delivered on Elekta linear accelerators.

Results: Comparison of VMAT and IMRT plans within the same patient and institute showed significantly better sparing for almost all OARs with VMAT. The average mean dose to the parotid glands and oral cavity was reduced from 27.2 Gy and 39.4 Gy for IMRT to 25.0 Gy and 36.7 Gy for VMAT, respectively. The dose conformity at 95\% of the prescribed dose for PTV boost and PTV total was 1.45 and 1.62 for IMRT and 1.37 and 1.50 for VMAT, respectively. The average effective delivery time was reduced from 13:15 min for IMRT to 5:54 min for VMAT.
\end{abstract}

Conclusions: Independently of institution-specific optimization strategies, the quality of the VMAT plans including double arcs was superior to step-and-shoot IMRT plans including 5-9 beam ports, while the effective treatment delivery time was shortened by $~ 50 \%$ with VMAT.

Keywords: Head-and-neck cancer, Simultaneous integrated boost, Volumetric modulated arc therapy, Intensity-modulated radiation therapy, Multi-institutional collaboration, SmartArc

\section{Background}

A notable difficulty with irradiation of head-and-neck cancer $(\mathrm{HNC})$ is the large number of organs at risk (OARs) in close proximity to regions with disease, including the salivary glands, spinal cord and brainstem, larynx and pharyngeal constrictors, oral mucosae, tongue and lips, masseter as well as eyes and inner ears. The challenging task for the treatment planner is to find the most optimal

\footnotetext{
* Correspondence: C.v.Vliet@nki.nl

'Department of Radiation Oncology, The Netherlands Cancer Institute Antoni van Leeuwenhoek Hospital, Plesmanlaan 121, Amsterdam, CX 1066, The Netherlands

Full list of author information is available at the end of the article
}

trade-off in sparing the different OARs for each individual patient. Often better sparing of one OAR implies sacrificing another OAR, and in most patients high-grade radiation-induced toxicity is unavoidable while ensuring sufficient dose coverage of the planning target volume (PTV). This may result in severe consequences for the quality of life of these patients.

Introduction of intensity-modulated radiation therapy (IMRT) techniques for the treatment of $\mathrm{HNC}$ replaced conventional 3D-conformal radiation therapy (3D-CRT) techniques, which resulted in much better dose conformity and sparing of the OARs and, therefore, less radiation-induced toxicity [1-3]. When using IMRT for

\section{Biomed Central}


irradiation of oropharyngeal cancer salivary function was less impaired, but the majority of the patients still suffered from some degree of xerostomia [4-6]. Braam et al. showed that the normal tissue complication probability (NTCP) at several time points after radiation therapy was less than $20 \%$ only if the mean dose to the parotid glands was lower than 25 Gy [7], a dose level that even with IMRT is often not achieved.

Recently, the next generation of IMRT techniques, volumetric modulated arc therapy (VMAT) has become widely available. Compared to static-beam IMRT, rotational VMAT is supposed to decrease treatment delivery times with at least similar or even better plan quality [8]. A number of single-institution studies have been published for VMAT for HNC [9-15]. The authors of these studies observed comparable or better PTV coverage and conformity as well as better sparing of OARs for VMAT compared to IMRT, while delivery times were shortened by $35-60 \%$. Recently, a multi-institutional study comparing different treatment technologies planned in different institutes has been reported [16]. However, no multi-institutional studies comparing VMAT vs. IMRT planned by multiple institutes using the same set of patients and treatment planning engines have been reported so far.

The here presented study is the result of a collaboration of five institutes in the Netherlands with similar equipment. This collaboration aimed at a fast and safe clinical introduction of VMAT for HNC (implemented under the name SmartArc and Oncentra VMAT in the treatment planning systems Pinnacle and Oncentra Masterplan, respectively) on Elekta equipment and a high quality of VMAT treatment planning in all participating institutes. To evaluate the potential of VMAT all five participating institutes generated IMRT and VMAT plans based on their locally developed IMRT planning knowledge. The data retrieved from this multi-institutional collaboration gives the possibility of a treatment planner-independent comparison of VMAT vs. step-and-shoot IMRT for HNC. The reason for this is that each institute uses different optimization strategies and sets of objectives, and has different preferences for sparing OARs while identical patient data was used for treatment planning.

\section{Methods}

\section{Patient selection and contouring}

For this retrospective treatment planning study, computed tomography $(\mathrm{CT})$ data including contouring of five patients with oropharyngeal cancer were selected (patient characteristics listed in Table 1). These patients were previously treated with the standard clinical protocols. According to the guidelines of the participating institutes, the patient data was properly anonimized and no informed consent of the patient was required. The planning target volumes (PTVs) and organs at risk (OARs), amongst others parotid glands, submandibular glands, oral cavity, larynx, pharyngeal constrictors and mandible, and if applicable, their corresponding substructures, were delineated by an experienced clinician. Delineation of an OAR was skipped in cases where the PTV completely encompassed this (sub-) structure.

\section{Dose prescription and plan acceptance parameters}

Both VMAT and IMRT plans were generated for a treatment in 32 fractions, to deliver a total dose of 56 Gy to the $\mathrm{PTV}_{\text {elective }}$ (i.e. a fraction dose of $1.75 \mathrm{~Gy}$ ) and a simultaneous integrated boost (SIB) to 69.12 Gy to regions with macroscopic disease (i.e. a fraction dose of 2.16 Gy). Primary goal of treatment planning was to cover at least 99\% of the volume of PTV elective and $\mathrm{PTV}_{\text {boost }}$ with $95 \%$ of the prescribed dose (53.2 Gy and 65.66 Gy, respectively), and to restrict the volume receiving more than $107 \%$ in

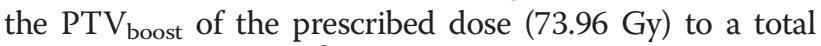
volume below $2 \mathrm{~cm}^{3}$ (all parameters summarized in Table 2). The maximal allowed point dose to OARs was 54 Gy for the brainstem and 50 Gy for the spinal cord. In addition, the mean dose preferably should be below 25 Gy for the parotid glands (for at least one parotid), submandibular glands and the oral cavity (if possible), and below $45 \mathrm{~Gy}$ for the larynx and pharyngeal constrictors.

\section{Treatment planning}

The CT data sets of five patients including contouring were shared between the participating institutes $(n=5)$. All institutes used their locally developed treatment planning technique and inverse planning objectives

Table 1 Patient characteristics

\begin{tabular}{|c|c|c|c|c|c|}
\hline Patient & Location & Classification & $\begin{array}{l}\text { Lymph } \\
\text { nodes } \\
\text { involved }\end{array}$ & $\begin{array}{c}\text { PTV elective } \\
\text { volume }\left(\mathrm{cm}^{3}\right)\end{array}$ & $\begin{array}{c}\text { PTV }_{\text {boost }} \\
\text { volume }\left(\mathrm{cm}^{3}\right)\end{array}$ \\
\hline 1 & Base of tongue $\mathrm{R}$ & T1N2aM0 & $2 \& 3 R$ & 501 & 105 \\
\hline 2 & Tonsil R & $\mathrm{T} 2 \mathrm{~N} 2 \mathrm{CM} 0$ & 3 R \& 2 bilat & 621 & 234 \\
\hline 3 & Tonsil L & $\mathrm{T} 3 \mathrm{~N} 2 \mathrm{CM} 0$ & 1,2,3,4 bilat & 992 & 381 \\
\hline 4 & Base of tongue $\mathrm{R}$ & $\mathrm{T} 3 \mathrm{~N} 2 \mathrm{CM} 0$ & $1 b$ R \& 2 bilat & 836 & 422 \\
\hline 5 & Tonsil L & $\mathrm{T} 2 \mathrm{~N} 1 \mathrm{M} 0$ & $2 L$ & 571 & 146 \\
\hline Average & & & & 704 & 258 \\
\hline
\end{tabular}


Table 2 Treatment planning objectives

\begin{tabular}{|c|c|c|c|}
\hline Structure & Parameter & Objective & Priority \\
\hline \multirow[t]{3}{*}{$\overline{\text { PTV }}$ boost } & $\begin{array}{l}\text { Prescribed } \\
\text { dose }\end{array}$ & 69.12 Gy $(32 * 2.16$ Gy) & \\
\hline & $V_{99 \%}$ & $\begin{array}{l}>95 \% \text { of prescribed dose } \\
\text { (= } 65.66 \mathrm{~Gy})\end{array}$ & High \\
\hline & $\mathrm{D}_{107 \%}$ & $<2 \mathrm{~cm}^{3}$ & High \\
\hline \multirow[t]{2}{*}{ PTV elective } & $\begin{array}{l}\text { Prescribed } \\
\text { dose }\end{array}$ & 56 Gy $(32 * 1.75$ Gy) & \\
\hline & $V_{99 \%}$ & $\begin{array}{l}>95 \% \text { of prescribed dose } \\
\text { (= } 53.2 \mathrm{~Gy})\end{array}$ & High \\
\hline Spinal Cord & $D_{\max }$ & $<50 \mathrm{~Gy}$ & High \\
\hline Brainstem & $D_{\max }$ & $<54 \mathrm{~Gy}$ & High \\
\hline \multirow[t]{2}{*}{ Parotid glands } & $\mathrm{D}_{\text {mean }}$ & Preferably < 25 Gy & High-Medium \\
\hline & & $\begin{array}{l}\text { For at least the } \\
\text { contralateral gland }\end{array}$ & \\
\hline $\begin{array}{l}\text { Submandibular } \\
\text { glands }\end{array}$ & $D_{\text {mean }}$ & Preferably < 25 Gy & Medium \\
\hline Oral cavity & $D_{\text {mean }}$ & Preferably< 25 Gy & Medium \\
\hline Larynx & $D_{\text {mean }}$ & Preferably < 45 Gy & Medium-Low \\
\hline $\begin{array}{l}\text { Pharyngeal } \\
\text { constrictors }\end{array}$ & $D_{\text {mean }}$ & Preferably < 45 Gy & Medium-Low \\
\hline Other OARs & & & Low \\
\hline
\end{tabular}

previously used with IMRT as starting point. VMAT and IMRT plans were generated using Pinnacle ${ }^{3}$ v9.0 (Philips Healthcare, Best, The Netherlands) $(n=4)$ or alternatively Oncentra Masterplan (Nucletron, Veenendaal, The Netherlands) ( $n=1$, institute C), which were commissioned for treatment delivery using Elekta linear accelerators (Elekta Oncology, Crawley, United Kingdom) equipped with either standard MLCs with $1 \mathrm{~cm}$ leaves not allowing interdigitation $(n=4)$ or a beam modulator with $0.4 \mathrm{~cm}$ leaves allowing interdigitation $(n=1$, institute D).

Both treatment planning systems (TPSs) used in this study employ nearly identical optimization modules developed by RaySearch Laboratories (RaySearch Laboratories, Stockholm, Sweden) for VMAT planning: p-RayArc marketed as SmartArc in Pinnacle ${ }^{3}$, and n-RayArc marketed as Oncentra VMAT in Oncentra Masterplan; and for IMRT planning: p-RayMachine marketed as Direct Machine Parameter Optimization (DMPO) in Pinnacle ${ }^{3}$, and n-RayMachine marketed as Direct Step and Shoot (DSS) in Oncentra Masterplan.

With VMAT and IMRT treatment planning, each optimization is started with generation of fluence maps. After typically $10-15$ iterations, at the so-called conversion iteration, segments are produced. Following an intermediate collapsed-cone dose calculation, optimization is continued using Direct Aperture Optimization (i.e., DMPO in Pinnacle ${ }^{3}$, and DSS in Oncentra Masterplan) up to a total of 30-50 iterations.

\section{IMRT planning parameters}

Step-and-shoot IMRT plans were generated according to each institute's practice using typically $5-9$ coplanar beams (one institute used one additional non-coplanar beam at $330^{\circ}$ at a couch angle of $90^{\circ}$ ) with a total of 50-70 segments. The lower limit for the segment size ranged from 4 to $9 \mathrm{~cm}^{2}$, with a minimum of 2 to 5 monitor units (MUs) per segment.

\section{VMAT planning parameters}

VMAT plans were generated using one dual arc (i.e. a double arc generated from a single arc at the segmentation step) with an arc length close to $360^{\circ}$. Before starting the study, all institutes had individually tested different arc setups with, for example, only a single arc or one or more dual arcs with varying arc lengths or combinations thereof. During discussion of the first results within the workgroup it was concluded that the use of a dual arc over the full range would yield the most promising, clinically acceptable results. The final resolution of control points within the arc was set to $4^{\circ}$, which was found to allow sufficient modulation at still acceptable duration of the optimization. The collimator angle was typically set to a value between $10^{\circ}$ and $30^{\circ}$ (or alternatively between $330^{\circ}$ and $350^{\circ}$ ) to avoid tongue-and-groove effects. All IMRT and VMAT plans were generated using $6 \mathrm{MV}$ photons.

VMAT plan optimization was restarted typically three to five times after the initial run without prior resetting of the earlier optimization result including segments and dose rate along the arcs (so-called "warm re-starts"). At the end of each of these optimization steps a full collapsed-cone dose calculation is performed. After a restart of the optimization altered dose distributions after adaptation of segments are calculated as a small perturbation on the previously calculated dose distribution using a simple pencil-beam based approach. This approximation leads to an accumulation of errors in the dose distribution during the optimization process, which is then "repaired" by the forced intermediate dose calculation after a fixed number of iterations. This procedure greatly improves accordance with the planning objectives in each step since dose distribution after full dose calculation including inhomogeneity corrections will be close to the altered dose distribution found during the optimization process.

If necessary and desirable, target doses and/or objective weights were adapted during the optimization process and in-between re-starts to further lower dose to OARs and/or improve PTV coverage. However, in case of large changes in the objectives the optimization was repeated including the segmentation step, i.e. re-started from scratch.

\section{Planning objectives}

Each institute started with their locally developed planning strategy including their specific set of objectives. 
After initial planning of one patient the planning procedures and parameters were discussed in the group and as a result, some institutes adjusted their individual planning parameters and set of objectives. Hereafter, each institute generated IMRT and VMAT plans for each of the five patients (including the initially planned patient if necessary) using a similar set of objectives for both IMRT and VMAT, with only small or no changes to objective doses and weights within the same patient.

A typical set of objectives to create a SIB plan always included for both $\mathrm{PTV}_{\text {elective }}$ and $\mathrm{PTV}_{\text {boost }}$ minimum and maximum dose objectives with high objective weights, i.e. 50-100 (if 100 would be the maximum for objective weights). In a limited number of institutes and cases, also uniform dose objectives were added. Furthermore, all institutes included maximum point dose objectives for spinal cord and brain stem with mostly intermediate objective weights, i.e. 5-50. Some institutes expanded these structures by $3-5 \mathrm{~mm}$ to create an extra safety margin, the so-called planning risk volume (PRV).

Dose to the other OARs including the left and right parotid, submandibular glands if delineated, oral cavity, larynx, pharyngeal constrictors and mandible was typically steered using maximum EUD objectives with mostly low objective weights, i.e. 1-10, and a gEUD factor of $\mathrm{a}=1$ (representing the mean dose). For each parotid, typically for the part of the parotid outside the PTV (or the PTV expanded by several $\mathrm{mm}$ ) at least one dose volume histogram (DVH) point objective was added with mostly low objective weights, i.e. 1-10. In addition, all institutes used ring structures to create steep dose gradients outside the $\mathrm{PTV}_{\text {elective }}$ and also between the two dose levels. Here, mostly maximum point dose objectives with low to intermediate objective weights, i.e. 1-20 were used. In addition, some of the participating institutes used dose objectives to restrict dose to one or more of the following OARs: lung tops, shoulders, eyes and inner ears.

\section{Determination of effective delivery times}

Delivery times for IMRT and VMAT plans were determined on Elekta linear accelerators without making use of the AFS (auto-field-setup) function to prepare beams and segments for delivery. Tests with enabled AFS yielded a time gain of 15 to 20 seconds per beam for IMRT, depending on the number of segments per beam; and for VMAT a gain of ca. 20 seconds in-between the two arcs. Therefore, if AFS is enabled the delivery times for IMRT reported in this study would be reduced by 1:30 to 3:00 min. It should be noted, however, that our timings neglect possible delays due to interaction with the patient in-between the delivery.

\section{Data retrieval and statistical analysis}

For the purpose of comparison, all participating institutes submitted their completed VMAT and IMRT plans in DICOM RTdose format. The dose distributions were imported into Pinnacle ${ }^{3}$ using an in-house developed import script, if necessary normalized to the minimal required PTV coverage, and DVHs and dose parameters were retrieved for each plan using automated procedures. The data from all five patients and five institutes was pooled by treatment modality and analyzed in SPSS v15.0 as a whole and, in addition, stratified by patient and by institute. Hypothesis testing was performed at 95\%

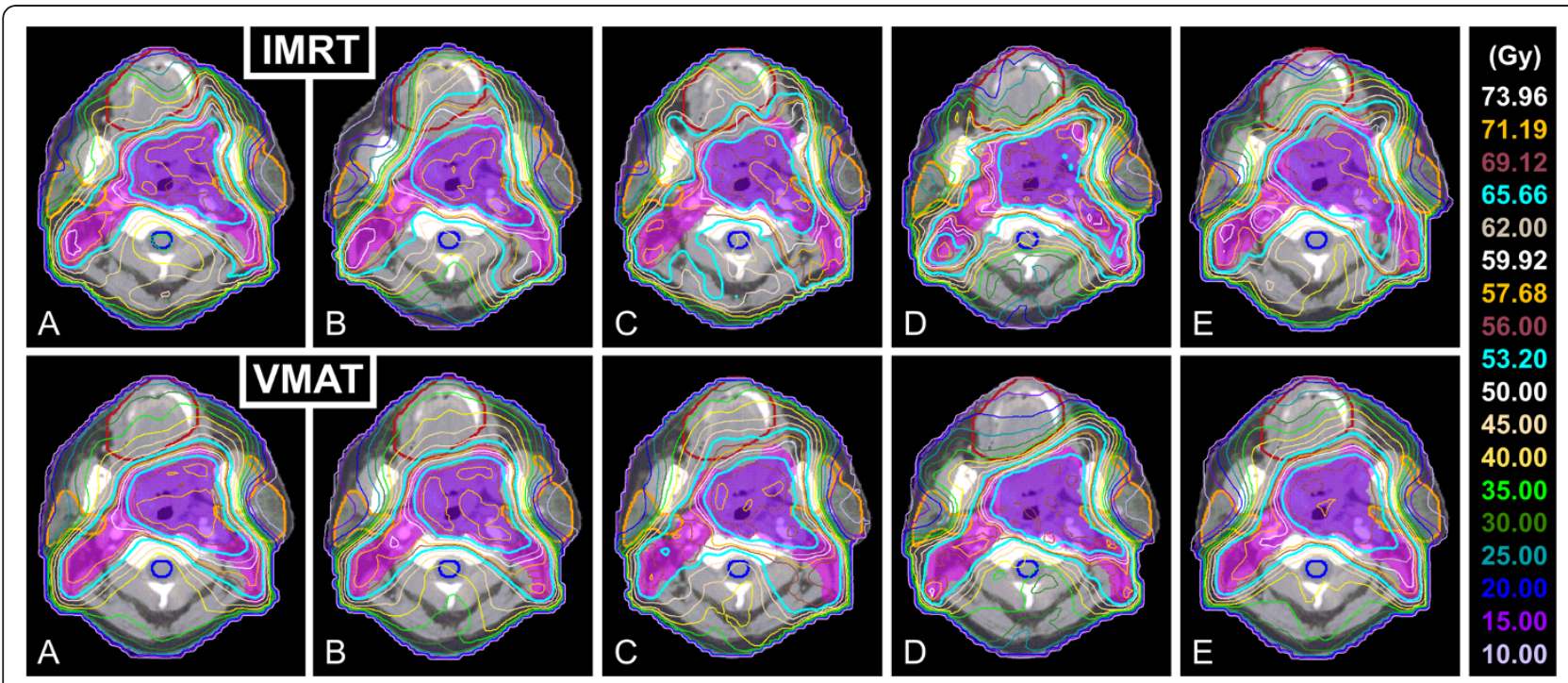

Figure 1 Dose distributions in a transverse slice for IMRT and VMAT plans of all participating institutes. Dose distributions in a transverse slice for IMRT and VMAT plans prepared by the participating institutes A to E. OARs are depicted with a thick solid line: oral cavity (brown), parotid glands (orange) and spinal cord (blue). 
confidence level using a paired two-sided Wilcoxon signed-rank test, if not otherwise specified.

\section{Results}

All institutes succeeded in producing clinically acceptable VMAT and IMRT plans for SIB treatments of HNC for all patients. All treatment planners aimed to limit dose to OARs as much as possible and tightened the objectives accordingly as long as the primary goals of the treatment planning protocol were still fulfilled (see Table 2 for a priority listing). Only small differences were found between IMRT plans from different institutes, which may be due to each institute's preferences and traditions in sparing the different OARs in daily clinical practice. Similar observations were made for the VMAT plans from the different institutes.

Dose distributions of IMRT and VMAT plans for a typical case prepared by the different institutes are shown in Figure 1. Generally, regardless of patient and institute, with VMAT the isodose surfaces encompassed the PTV tighter with similar or better sparing of the OARs. This was reflected in steeper dose fall-offs for the corresponding DVHs of the different PTVs (see Figure 2) and also smaller dose conformity indices (CIs) for VMAT plans. The $\mathrm{CI}_{95}$, defined by the ratio of total volume receiving $95 \%$ of the prescribed doses (i.e., 53.2 Gy and 65.66 Gy for PTV elective

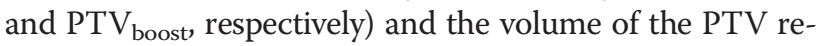
ceiving the same dose, was found to be significantly better with VMAT for both PTV boost and PTV IMRT for the pooled data (summarized in Table 3); as well when stratified by institute (see Table 4) or by patient (see Table 5). Comparison of the DVHs of different OARs reveals that with VMAT dose to OARs is in almost all cases reduced compared to IMRT (see Figure 3).

In addition, with VMAT the isodose surfaces encompassed the PTVs more smoothly (i.e. less occurrence of high dose bulges reaching far outside the PTVs) and fewer hot spots outside the PTVs were observed (see Figure 1 for a typical example). This was also reflected in reduced volumes of healthy tissue receiving doses above 30 Gy for VMAT compared to IMRT. This is illustrated by the average absolute volume difference between both techniques shown in Figure 4.

\section{VMAT vs. IMRT based on pooled data}

Comparison of the plan parameters for VMAT and IMRT plans generated for the same patient by the same institute showed significantly better sparing for most OARs (see Table 3). Figure 4 shows averaged DVHs for the pooled data for different OARs for VMAT (black solid line) and IMRT (black dashed line) and corresponding p-values (grey solid line). The average maximum point dose and $\mathrm{D}_{1 \%}$ (1\% of the volume receives more than

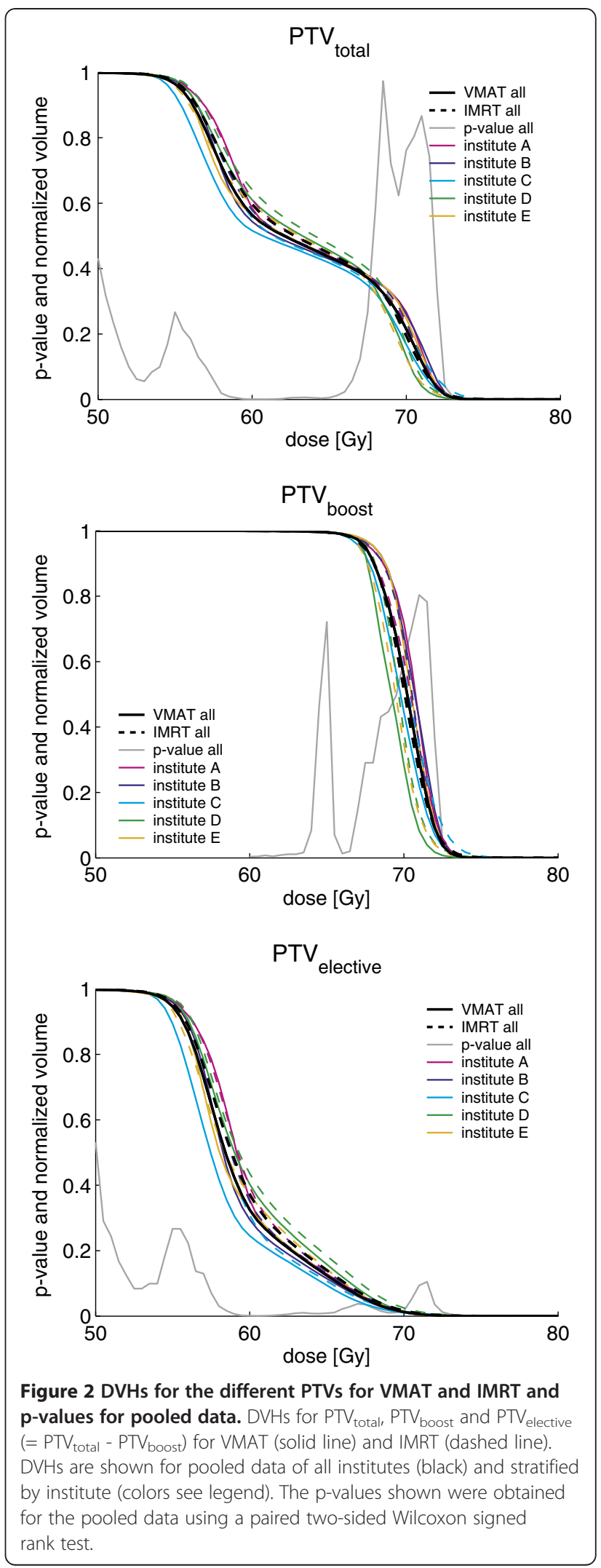


Table 3 Plan parameters and significance of differences based on the pooled data of five patients and five institutes

\begin{tabular}{|c|c|c|c|c|c|}
\hline & & $\begin{array}{c}\text { VMAT } \\
\text { average } \pm \\
1 \mathrm{SD}^{*}\end{array}$ & $\begin{array}{c}\text { IMRT } \\
\text { average } \pm \\
1 \mathrm{SD}^{*}\end{array}$ & $\begin{array}{c}\text { Average difference } \pm \\
1 \text { SD* } \\
\text { (VMAT - IMRT) }\end{array}$ & $\begin{array}{c}\text { p-value } \\
\text { (Wilcoxon's } \\
\text { signed-rank test)^ }\end{array}$ \\
\hline PTV $_{\text {boost }}$ & $\mathrm{Cl}_{95}$ & $1.37 \pm 0.08$ & $1.45 \pm 0.11$ & $-0.08 \pm 0.09$ & .001 \\
\hline PTV total & $\mathrm{Cl}_{95}$ & $1.50 \pm 0.09$ & $1.62 \pm 0.10$ & $-0.12 \pm 0.07$ & $<.001$ \\
\hline \multirow[t]{3}{*}{ Normal tissue } & $V_{5 G y}\left(\mathrm{~cm}^{3}\right)$ & $5050 \pm 730$ & $5030 \pm 750$ & $-20 \pm 260$ & n.s. \\
\hline & $V_{10 G y}\left(\mathrm{~cm}^{3}\right)$ & $4050 \pm 630$ & $3970 \pm 590$ & $-80 \pm 160$ & n.s. \\
\hline & $V_{20 G y}\left(\mathrm{~cm}^{3}\right)$ & $2830 \pm 510$ & $2860 \pm 480$ & $30 \pm 150$ & n.s. \\
\hline \multirow[t]{3}{*}{ Spinal cord } & $D_{\max }(G y)$ & $\mathbf{4 5 . 1} \pm 3.5$ & $46.6 \pm 3.0$ & $-1.5 \pm 2.3$ & .001 \\
\hline & $\mathrm{D}_{1 \%}(\mathrm{~Gy})$ & $43.4 \pm 3.7$ & $\mathbf{4 4 . 4} \pm 3.5$ & $-0.9 \pm 2.2$ & .005 \\
\hline & $D_{\text {mean }}(G y)$ & $29.3 \pm 4.4$ & $29.8 \pm 3.6$ & $-0.5 \pm 2.4$ & n.s. \\
\hline \multirow[t]{3}{*}{ Brain stem } & $\mathrm{D}_{\max }(\mathrm{Gy})$ & $46.4 \pm 5.4$ & $47.1 \pm 4.7$ & $-0.7 \pm 4.6$ & n.s. \\
\hline & $D_{1 \%}(G y)$ & $43.8 \pm 5.8$ & $44.0 \pm 5.5$ & $-0.2 \pm 4.9$ & n.s. \\
\hline & $D_{\text {mean }}(G y)$ & $13.6 \pm 3.6$ & $14.5 \pm 4.3$ & $-1.0 \pm 2.4$ & n.s. \\
\hline \multirow[t]{3}{*}{ Parotid gland ipsilateral } & $V_{25 G y}(\%)$ & $42.9 \pm 15.6$ & $50.3 \pm 20.0$ & $-7.4 \pm 14.0$ & $<.001$ \\
\hline & $\mathrm{V}_{39 \mathrm{~Gy}}(\%)$ & $30.0 \pm 14.6$ & $35.9 \pm 18.6$ & $-5.9 \pm 11.2$ & $<.001$ \\
\hline & $D_{\text {mean }}(G y)$ & $\mathbf{2 8 . 0} \pm 7.5$ & $31.1 \pm 9.1$ & $-3.1 \pm 5.1$ & $<.001$ \\
\hline \multirow[t]{3}{*}{ Parotid gland contralateral } & $V_{25 G y}(\%)$ & $31.0 \pm 7.1$ & $34.5 \pm 6.8$ & $-3.5 \pm 3.6$ & $<.001$ \\
\hline & $V_{39 G y}(\%)$ & $18.3 \pm 6.0$ & $20.8 \pm 6.1$ & $-2.5 \pm 2.5$ & $<.001$ \\
\hline & $D_{\text {mean }}(G y)$ & $22.0 \pm 2.9$ & $23.3 \pm 2.8$ & $-1.3 \pm 1.5$ & $<.001$ \\
\hline \multirow[t]{3}{*}{ Submandibular gland contralateral ${ }^{\$}$} & $\mathrm{~V}_{39 \mathrm{~Gy}}(\%)$ & $88.1 \pm 15.3$ & $90.8 \pm 13.5$ & $-2.7 \pm 6.9$ & n.s. \\
\hline & $V_{60 G y}(\%)$ & $\mathbf{1 6 . 4} \pm 21.4$ & $21.8 \pm 27.3$ & $-5.5 \pm 8.5$ & .020 \\
\hline & $D_{\text {mean }}(G y)$ & $53.0 \pm 5.9$ & $54.2 \pm 6.1$ & $-1.3 \pm 1.8$ & .027 \\
\hline \multirow[t]{3}{*}{ Oral cavity } & $V_{25 G y}(\%)$ & $79.8 \pm 22.9$ & $86.3 \pm 15.7$ & $-6.5 \pm 10.2$ & .011 \\
\hline & $\mathrm{V}_{39 \mathrm{~Gy}}(\%)$ & $\mathbf{4 0 . 6} \pm 22.0$ & $\mathbf{4 8 . 8} \pm 23.3$ & $-8.2 \pm 12.4$ & .002 \\
\hline & $\mathrm{D}_{\text {mean }}(\mathrm{Gy})$ & $36.7 \pm 7.8$ & $39.4 \pm 7.3$ & $-2.7 \pm 2.8$ & $<.001$ \\
\hline \multirow[t]{3}{*}{ Larynx } & $V_{39 G y}(\%)$ & $75.4 \pm 24.7$ & $79.1 \pm 21.6$ & $-3.8 \pm 6.7$ & .012 \\
\hline & $V_{45 G y}(\%)$ & $54.1 \pm 27.4$ & $58.2 \pm 26.5$ & $-4.1 \pm 9.3$ & n.s. \\
\hline & $D_{\text {mean }}(G y)$ & $\mathbf{4 5 . 5} \pm 5.3$ & $46.5 \pm 4.4$ & $-1.0 \pm 1.5$ & .004 \\
\hline \multirow[t]{3}{*}{ Pharyngeal constrictors ${ }^{\circ}$} & $V_{39 G y}(\%)$ & $78.7 \pm 24.5$ & $81.5 \pm 20.0$ & $-2.8 \pm 15.2$ & n.s. \\
\hline & $V_{45 G y}(\%)$ & $59.7 \pm 29.4$ & $57.5 \pm 25.5$ & $2.2 \pm 19.9$ & n.s. \\
\hline & $D_{\text {mean }}(G y)$ & $47.1 \pm 5.3$ & $46.9 \pm 3.8$ & $0.2 \pm 2.7$ & n.s. \\
\hline \multirow[t]{3}{*}{ Mandible $^{\circ}$} & $\mathrm{V}_{39 \mathrm{~Gy}}(\%)$ & $74.0 \pm 13.1$ & $78.2 \pm 11.8$ & $-4.2 \pm 8.4$ & .025 \\
\hline & $V_{60 G y}(\%)$ & $25.7 \pm 15.0$ & $30.1 \pm 16.2$ & $-4.4 \pm 5.1$ & $<.001$ \\
\hline & $D_{\text {mean }}(G y)$ & $\mathbf{4 8 . 6} \pm 5.5$ & $50.3 \pm 5.7$ & $-1.7 \pm 1.7$ & $<.001$ \\
\hline Effective delivery time ${ }^{\#}$ & (min:sec) & $\mathbf{5 : 5 4} \pm 1: 05$ & $13: 15 \pm 1: 38$ & $-7: 21 \pm 1: 55$ & $<.001$ \\
\hline MUs & - & $643 \pm 111$ & $\mathbf{8 2 8} \pm 149$ & $-185 \pm 129$ & $<.001$ \\
\hline
\end{tabular}

* Standard deviations (SDs) are given as absolute values.

$\wedge$ Hypothesis testing using a paired t-test results in almost identical significances at $95 \%$ confidence level.

${ }^{\$}$ The contralateral submandibular gland was delineated in only three patients.

- Not all institutes used objectives to control dose for these structures.

\# Based on effective delivery times reported by four institutes.

this dose) to spinal cord and brain stem were lower with VMAT, though only for the spinal cord at significant level. With VMAT we observed a significantly lower average mean dose and $\mathrm{V}_{39 \mathrm{~Gy}}$ (i.e. the percentage of volume receiving more than $39 \mathrm{~Gy}$ ) for ipsilateral and contralateral parotid glands, mandible, oral cavity and larynx. In addition, the $V_{25}$ Gy was significantly improved for the parotid glands and the oral cavity. The contralateral submandibular gland was delineated in only three out of the five patients and was always located in very close proximity to the PTV. For this reason, we generally observed high doses to the contralateral submandibular gland with both 
Table 4 Plan parameters stratified by institute

\begin{tabular}{|c|c|c|c|c|c|c|c|}
\hline & & $\begin{array}{l}\text { Institute } \\
\text { Technique }\end{array}$ & A & B & C & D & $\mathrm{E}$ \\
\hline \multirow[t]{2}{*}{$\mathrm{PTV}_{\text {boost }}$} & \multirow[t]{2}{*}{$\mathrm{Cl}_{95}$} & VMAT & 1.39 & 1.35 & 1.31 & 1.43 & 1.38 \\
\hline & & IMRT & 1.43 & 1.42 & 1.37 & 1.55 & 1.47 \\
\hline \multirow[t]{2}{*}{ PTV } & \multirow[t]{2}{*}{$\mathrm{Cl}_{95}$} & VMAT & 1.51 & 1.49 & 1.54 & 1.45 & 1.51 \\
\hline & & IIVIn! & 1.58 & 1.63 & 1.70 & 1.55 & 1.65 \\
\hline \multirow[t]{2}{*}{ Spinal cord } & \multirow{2}{*}{$\begin{array}{l}D_{1 \%} \\
(G y)\end{array}$} & VMAT & 39.1 & 45.6 & 47.7 & 41.1 & 43.8 \\
\hline & & IMRT & 39.7 & 46.7 & 48.0 & 42.0 & 45.5 \\
\hline \multirow[t]{2}{*}{ Brain stem } & \multirow{2}{*}{$\begin{array}{l}D_{1 \%} \\
(G y)\end{array}$} & VMAT & 44.1 & 45.6 & 49.4 & 35.4 & 44.6 \\
\hline & & IMRT & 42.9 & 45.8 & 46.9 & 40.0 & 44.4 \\
\hline \multirow{2}{*}{$\begin{array}{l}\text { Parotid gland } \\
\text { ipsilateral }\end{array}$} & \multirow{2}{*}{$\begin{array}{c}D_{\text {mean }} \\
(\mathrm{Gy})\end{array}$} & VMAT & 27.6 & 26.6 & 33.3 & 23.2 & 29.3 \\
\hline & & ART & 28.5 & 28.4 & 34.2 & 25.3 & 39.0 \\
\hline \multirow{2}{*}{$\begin{array}{l}\text { Parotid gland } \\
\text { contralateral }\end{array}$} & \multirow{2}{*}{$\begin{array}{c}D_{\text {mean }} \\
(\mathrm{Gy})\end{array}$} & VMAT & 23.9 & 21.1 & 23.4 & 20.6 & 20.8 \\
\hline & & IMRT & 24.8 & 22.7 & 23.6 & 21.5 & 23.6 \\
\hline \multirow{2}{*}{$\begin{array}{l}\text { Submandibular } \\
\text { gland } \\
\text { contralateral }\end{array}$} & \multirow{2}{*}{$\begin{array}{c}D_{\text {mean }} \\
(\mathrm{Gy})\end{array}$} & VMAT & 49.4 & 58.0 & 57.4 & 47.5 & 52.6 \\
\hline & & IMRT & 49.1 & 58.6 & 58.1 & 51.1 & 54.3 \\
\hline \multirow[t]{2}{*}{ Oral cavity } & \multirow{2}{*}{$\begin{array}{c}\mathrm{D}_{\text {mean }} \\
(\mathrm{Gy})\end{array}$} & VMAT & 40.0 & 35.2 & 41.6 & 30.7 & 36.0 \\
\hline & & in & 42.5 & 40.2 & 43.6 & 33.5 & 37.4 \\
\hline \multirow[t]{2}{*}{ Larynx } & \multirow{2}{*}{$\begin{array}{c}D_{\text {mean }} \\
(\mathrm{Gy})\end{array}$} & VMAT & 51.4 & 44.5 & 49.4 & 43.5 & 38.6 \\
\hline & & IMRT & 51.5 & 46.3 & 49.1 & 44.5 & 40.9 \\
\hline \multirow{2}{*}{$\begin{array}{l}\text { Pharyngeal } \\
\text { constrictors }\end{array}$} & \multirow{2}{*}{$\begin{array}{c}\mathrm{D}_{\text {mean }} \\
(\mathrm{Gy})\end{array}$} & VMAT & 52.0 & 45.0 & 49.8 & 43.9 & 44.7 \\
\hline & & IMRT & 49.0 & 45.7 & 48.8 & 44.2 & 46. \\
\hline \multirow[t]{2}{*}{ Mandible ${ }^{\circ}$} & \multirow{2}{*}{$\begin{array}{c}\mathrm{D}_{\text {mean }} \\
(\mathrm{Gy})\end{array}$} & VMAT & 48.8 & 48.0 & 50.5 & 47.6 & 48.0 \\
\hline & & IMRT & 49.7 & 49.9 & 51.1 & 49.3 & 51.3 \\
\hline \multirow{2}{*}{$\begin{array}{l}\text { Effective delivery } \\
\text { time }\end{array}$} & \multirow{2}{*}{$\begin{array}{l}\text { (min: } \\
\text { sec) }\end{array}$} & VMAT & $4: 51$ & $6: 50$ & - & $5: 15$ & $6: 4$ \\
\hline & & IMRT & $11: 47$ & $12: 08$ & - & $15: 04$ & $14: 00$ \\
\hline \multirow[t]{2}{*}{ MUs } & \multirow[t]{2}{*}{-} & VMAT & 600 & 602 & 540 & 774 & 698 \\
\hline & & IMRT & 830 & 670 & 824 & 841 & 975 \\
\hline
\end{tabular}

Pairs in bold denote statistically significant differences between VMAT and IMRT.

Pairs in italic denote that the averaged plan parameter is better for IMRT.

- Not all institutes used objectives to control dose for these structures.

VMAT and IMRT (see DVHs in Figure 3) and the plan acceptance parameters could not be fulfilled in any case.

VMAT delivery times estimated by the TPSs for both arcs in the dual arc were on average 3:56 min and ranged from 3:00 to 5:43 min, depending on each institute's practice and difficulty of the case. In almost all cases, the TPS made use of the maximum delivery time allocated by the planner. The effective delivery times for VMAT (defined as the time from start of the first arc and end of the second arc) based on reports from four institutes were on average 5:54 $\mathrm{min}$ and ranged from 4:18 to 7:57 $\mathrm{min}$. One institute could not report effective delivery times for their VMAT plans due to a delay with installing VMAT licenses for their treatment machines. The delivery times of the IMRT plans (defined from start of the first segment of the first beam till end of the last segment of the last beam) were on average 13:15 min and ranged from 9:40 to 15:56 $\mathrm{min}$.
Based on these results, with VMAT for HNC the delivery times can be expected to be reduced by $\sim 50 \%$ compared to IMRT.

\section{Evaluation per institute}

When stratifying the data by institute the overall picture regarding improved plan parameters with VMAT remained the same. For all plan parameters listed in Table 4 with exception of the contralateral submandibular gland, larynx and brain stem VMAT yielded better results, regardless of institute, though not always at statistically significant level. Please note that statistical power of the stratified comparison is low since the statistical analysis is based on only five pairs. However, the mean dose to the ipsilateral parotid gland was in all cases significantly better for VMAT, and for most institutes this also held for the contralateral parotid gland.

The data listed in Table 4 also provides interesting information on each institute's planning preferences. For institute $\mathrm{C}$ dose conformity to the $\mathrm{PTV}_{\text {boost }}$ had probably a relative high priority compared to other institutes, and sparing of the ipsilateral parotid gland had lower priority, as well as achieving low maximal doses to spinal cord and brain stem. Institute A seemed to give high preference to an as low as possible maximal point dose in spinal cord and brain stem, whereas institutes B, D and E probably tried to achieve a as low as possible mean dose to the contralateral parotid gland, and institute D even tried this for the ipsilateral parotid gland. Probably, institute $\mathrm{D}$ profitted here from using a beam modulator that supports interdigitation of the MLC leaves, which allows a higher degree of dose modulation.

Institute $\mathrm{D}$ also seemed to pay increased attention to low doses to the oral cavity while dose conformity to the PTVs seemed to play a less important role. These findings were also supported by the underlying dosimetric data of the individual patients, where almost all respective pairings of dosimetric parameters showed the same preferences as the averages per institute listed in Table 4. This supports our idea that even within quite defined boundary conditions for treatment planning, including a beforehand agreed treatment protocol and guidelines for sparing OARs, quite some variation is possible in the results of treatment planning studies like the one presented here.

\section{Evaluation per patient}

Likewise, stratification of the data by patient does not largely change the previous findings. All plan parameters except the mean doses to the larynx were lower for VMAT compared to IMRT, though not all at significant level (see Table 5). A reason for this observation could be that the larynx is located in-between the two sides of

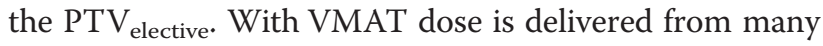


Table 5 Plan parameters stratified by patient

\begin{tabular}{|c|c|c|c|c|c|c|c|}
\hline & & $\begin{array}{c}\text { Patient } \\
\text { Technique }\end{array}$ & 1 & 2 & 3 & 4 & 5 \\
\hline \multirow[t]{2}{*}{ PTV boost } & $\mathrm{Cl}_{95}$ & VMAT & $1.37 \pm 0.03$ & $1.41 \pm 0.06$ & $1.46 \pm 0.10$ & $1.30 \pm 0.03$ & $1.31 \pm 0.03$ \\
\hline & & IMRT & $1.54 \pm 0.09$ & $1.44 \pm 0.04$ & $1.50 \pm 0.07$ & $1.33 \pm 0.05$ & $1.42 \pm 0.17$ \\
\hline \multirow[t]{2}{*}{ PTV total } & $\mathrm{Cl}_{95}$ & VMAT & $1.43 \pm 0.06$ & $1.58 \pm 0.07$ & $1.52 \pm 0.04$ & $1.57 \pm 0.08$ & $1.40 \pm 0.04$ \\
\hline & & IMRT & $1.53 \pm 0.04$ & $1.71 \pm 0.09$ & $1.63 \pm 0.06$ & $1.67 \pm 0.10$ & $1.57 \pm 0.11$ \\
\hline \multirow[t]{2}{*}{ Spinal cord } & $D_{1 \%}(G y)$ & VMAT & $\mathbf{4 2 . 1} \pm 5.5$ & $44.7 \pm 2.6$ & $43.4 \pm 3.5$ & $44.0 \pm 4.1$ & $42.9 \pm 3.6$ \\
\hline & & IMRT & $43.8 \pm 4.3$ & $44.3 \pm 2.9$ & $44.8 \pm 2.4$ & $44.5 \pm 4.7$ & $44.4 \pm 4.2$ \\
\hline \multirow[t]{2}{*}{ Brain stem } & $D_{1 \%}(G y)$ & VMAT & $48.3 \pm 4.7$ & $42.1 \pm 6.0$ & $45.5 \pm 5.6$ & $41.4 \pm 6.9$ & $41.7 \pm 5.0$ \\
\hline & & IMRT & $48.2 \pm 1.9$ & $45.7 \pm 4.3$ & $47.6 \pm 4.2$ & $40.5 \pm 3.6$ & $37.9 \pm 5.0$ \\
\hline \multirow[t]{2}{*}{ Parotid gland ipsilateral } & $D_{\text {mean }}(G y)$ & VMAT & $21.7 \pm 2.0$ & $\mathbf{3 0 . 3} \pm 4.9$ & $\mathbf{2 4 . 8} \pm 3.5$ & $\mathbf{3 8 . 1} \pm 9.2$ & $25.1 \pm 2.4$ \\
\hline & & IMRT & $23.6 \pm 1.7$ & $33.3 \pm 3.5$ & $\mathbf{3 0 . 5} \pm 10.2$ & $\mathbf{4 1 . 8} \pm 11.0$ & $26.3 \pm 3.2$ \\
\hline \multirow[t]{2}{*}{ Parotid gland contralateral } & $D_{\text {mean }}(G y)$ & VMAT & $\mathbf{2 1 . 1} \pm 1.9$ & $23.1 \pm 1.0$ & $22.1 \pm 2.1$ & $25.0 \pm 2.9$ & $\mathbf{1 8 . 6} \pm 1.9$ \\
\hline & & IMRT & $23.2 \pm 1.9$ & $\mathbf{2 4 . 1} \pm 1.3$ & $24.2 \pm 0.5$ & $25.3 \pm 3.5$ & $\mathbf{1 9 . 4} \pm 1.5$ \\
\hline \multirow[t]{2}{*}{ Submandibular gland contralateral } & $D_{\text {mean }}(G y)$ & VMAT & $49.2 \pm 6.0$ & $55.0 \pm 6.3$ & - & - & $54.8 \pm 4.3$ \\
\hline & & IMRT & $49.6 \pm 5.6$ & $\mathbf{5 7 . 4} \pm 6.4$ & - & - & $55.7 \pm 3.7$ \\
\hline \multirow[t]{2}{*}{ Oral cavity } & $D_{\text {mean }}(G y)$ & VMAT & $31.3 \pm 4.8$ & $34.7 \pm 5.4$ & $33.3 \pm 7.0$ & $47.7 \pm 1.7$ & $36.5 \pm 7.4$ \\
\hline & & IMRT & $\mathbf{3 4 . 2} \pm 4.8$ & $38.2 \pm 3.1$ & $\mathbf{3 7 . 4} \pm 8.9$ & $48.8 \pm 1.7$ & $38.7 \pm 7.2$ \\
\hline \multirow[t]{2}{*}{ Larynx } & $D_{\text {mean }}(G y)$ & VMAT & $43.2 \pm 4.4$ & $44.9 \pm 7.3$ & $47.1 \pm 4.6$ & $46.8 \pm 5.5$ & $45.3 \pm 5.5$ \\
\hline & & IMRT & $44.5 \pm 4.3$ & $45.5 \pm 5.8$ & $47.5 \pm 4.2$ & $47.7 \pm 4.7$ & $47.0 \pm 3.9$ \\
\hline \multirow[t]{2}{*}{ Pharyngeal constrictors ${ }^{\circ}$} & $D_{\text {mean }}(G y)$ & VMAT & $42.3 \pm 3.9$ & $50.5 \pm 6.6$ & $48.9 \pm 2.6$ & $48.0 \pm 4.9$ & $45.7 \pm 5.2$ \\
\hline & & IMRT & $42.1 \pm 2.7$ & $48.2 \pm 3.7$ & $49.3 \pm 2.3$ & $47.9 \pm 3.6$ & $46.9 \pm 2.6$ \\
\hline \multirow[t]{2}{*}{ Mandible $^{\circ}$} & $D_{\text {mean }}(G y)$ & VMAT & $41.7 \pm 1.3$ & $45.1 \pm 1.4$ & $55.2 \pm 1.7$ & $54.2 \pm 1.5$ & $46.7 \pm 1.7$ \\
\hline & & IMRT & $42.4 \pm 0.9$ & $47.2 \pm 1.2$ & $57.6 \pm 1.5$ & $55.2 \pm 1.6$ & $48.9 \pm 1.7$ \\
\hline \multirow[t]{2}{*}{ Effective delivery time } & (min:sec) & VMAT & $5: 38 \pm 0: 42$ & $6: 00 \pm 0: 48$ & $6: 22 \pm 1: 28$ & $6: 07 \pm 1: 31$ & $5: 26 \pm 1: 04$ \\
\hline & & IMRT & $12: 52 \pm 0: 41$ & $13: 09 \pm 2: 18$ & $13: 46 \pm 1: 07$ & $13: 30 \pm 1: 50$ & $12: 57 \pm 2: 23$ \\
\hline \multirow[t]{2}{*}{ MUs } & - & VMAT & $\mathbf{5 8 1} \pm 55$ & $641 \pm 90$ & $735 \pm 170$ & $668 \pm 91$ & $589 \pm 73$ \\
\hline & & IMRT & $702 \pm 92$ & $836 \pm 166$ & $975 \pm 125$ & $855 \pm 100$ & $773 \pm 142$ \\
\hline
\end{tabular}

Pairs in bold denote statistically significant differences between VMAT and IMRT.

Pairs in italic denote that the averaged plan parameter is better for IMRT.

- Not all institutes used objectives to control dose for these structures.

directions and therefore more smeared out on OARs in such constellations; whereas with IMRT large parts of the dose are often given from directions omitting the OARs in-between the PTV.

\section{Discussion}

Analysis of the data resulting from this multi-institutional collaboration on the clinical introduction of VMAT for HNC shows that VMAT plans including a double arc generated with Pinnacle ${ }^{3}$ or Oncentra Masterplan have an improved plan quality compared to IMRT plans with 5-9 beam ports. This observation is valid independently of institution-specific planning strategies, choice of the set of objectives, or preferences for sparing OARs. Based on the effective delivery times reported from four institutes we expect a reduction in the effective delivery times of 50\% with VMAT compared to IMRT. The decreased treatment delivery time obtained with VMAT will improve patient comfort and result in a smaller impact of intra-fraction movements, as described by Hoogeman et al. [17].

Several single-institution studies comparing VMAT and IMRT for HNC have been reported in literature, most based on RapidArc [9,10] and on SmartArc [11-15]. The strength of the study presented here bases on its multiinstitutional setup and the rather limited regulations regarding the planning environment and procedures, resulting in varying solutions for the same set of patients. All plans were generated using planning environment and procedures that were (or will shortly be) adopted for routine clinical use. As such, the data presented in this study reflects a broad range of clinically achievable and acceptable results and, therefore, allows a planner-independent evaluation of the potential of VMAT vs. IMRT for irradiation of HNC. 

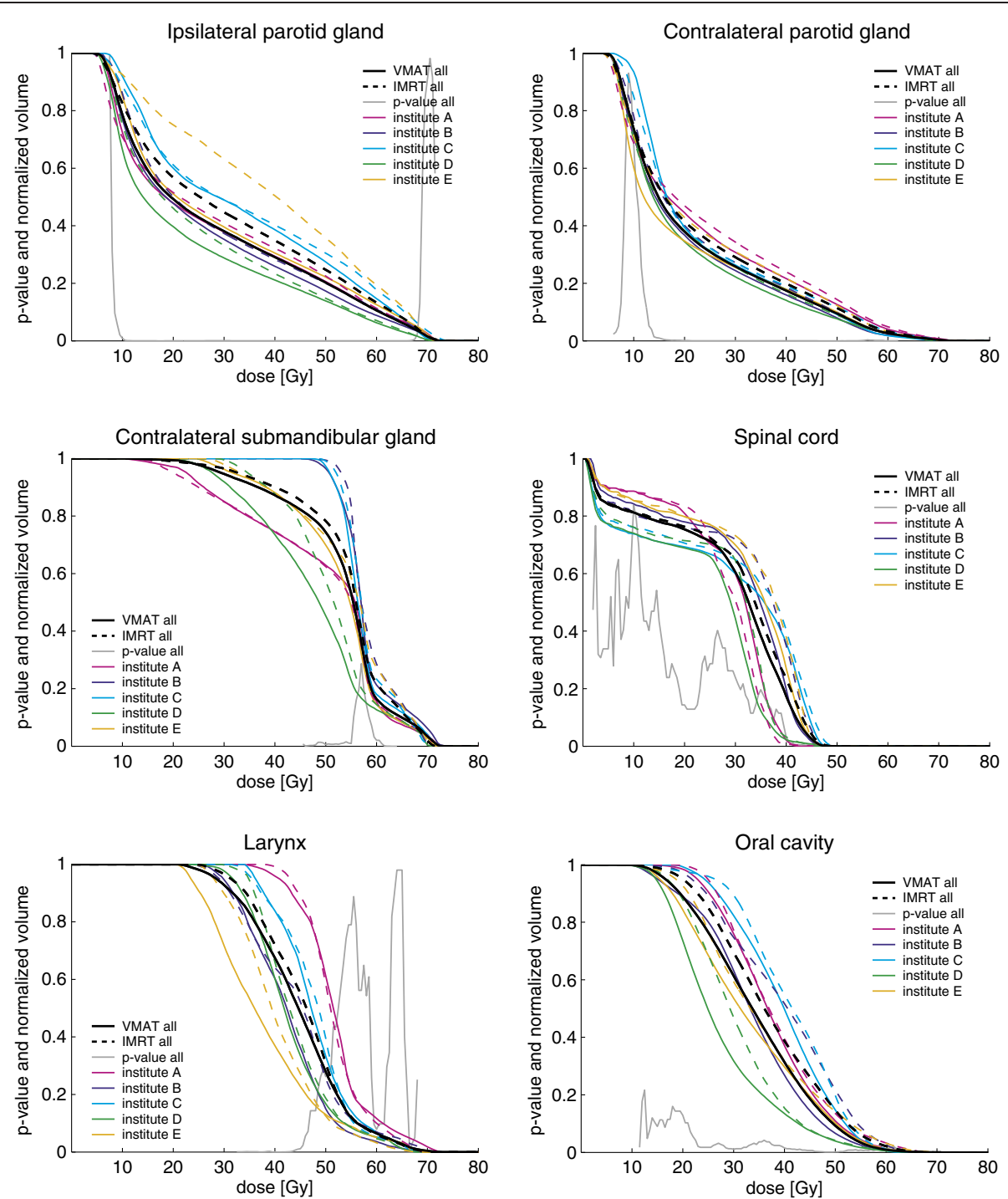

Figure 3 DVHs for different OARs for VMAT and IMRT and p-value for pooled data. DVHs for parotid and submandibular glands, spinal cord, larynx and oral cavity for VMAT (solid line) and IMRT (dashed line). DVHs are shown for pooled data of all institutes (black) and stratified by institute (colors see legend). The p-values shown were obtained for the pooled data using a paired two-sided Wilcoxon signed rank test.

IMRT has been the standard for radiation therapy of advanced $\mathrm{HNC}$ in all participating institutes for several years by now, and consequently, all institutes had a similar level of experience in IMRT treatment planning when starting this study. All participating institutes performed IMRT and VMAT treatment planning using the same CT data sets including contouring, ensuring excellent comparability of the data between institutes. The TPSs employed in this study, Pinnacle ${ }^{3}$ and Oncentra, both make use of nearly identical VMAT and IMRT optimization modules developed by RaySearch Laboratories. Therefore, the data retrieved from these two TPSs was pooled and analyzed as one population.
Pair-wise analysis of VMAT and IMRT plans within the same patient and institute showed for almost all plan pairs better sparing of OARs and dose conformity with VMAT (please note that statistical power is low in the stratified comparison). A major reason for this could be the rotational character of VMAT which allows dose delivery from many more directions than with static-beam IMRT with mostly 5-9 beam ports used. With VMAT optimization dose is automatically redistributed along the arc, which means that to a certain extent beam angle optimization is inherent to VMAT. The additional degrees of freedom with VMAT lead to the better dose conformity, which in turn allows for a better sparing of OARs in close proximity to the PTVs. 


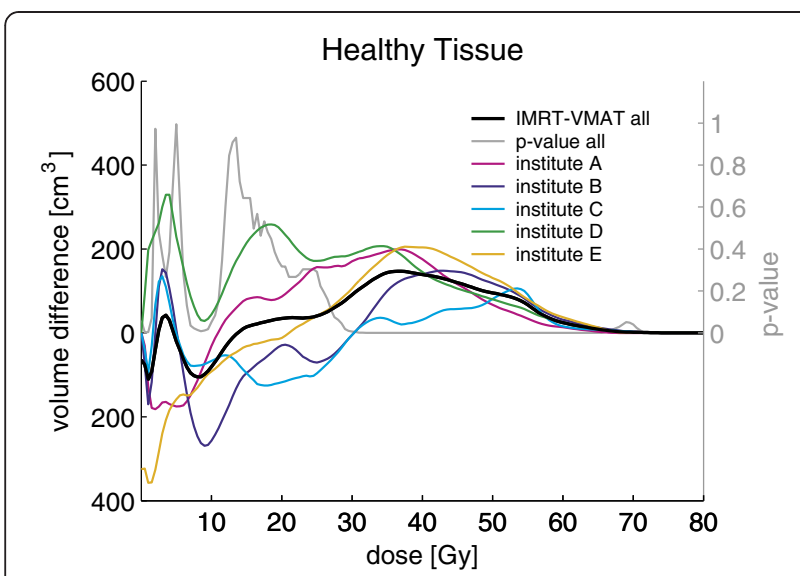

Figure 4 Averaged absolute volume difference between DVHs for healthy tissue and $\mathrm{p}$-value for pooled data. Averaged absolute volume difference between DVHs for IMRT and VMAT for healthy tissue for the pooled data (black) and stratified by institute (colors see legend). The $p$-values shown were obtained for the pooled data using a paired two-sided Wilcoxon signed rank test.

Further exploration of the underlying dosimetric data yielded that one institute chose to completely sacrifice one of the parotid glands with IMRT for some patients, but was able to spare it quite successfully with VMAT. Already in 1996, Eisbruch et al. reported on a 3D-CRT technique to spare the contralateral parotid gland, while deliberately accepting underdosage in the surrounding target volume with supposedly "lower" risk to contain disease [18]. With the introduction of IMRT, this technique was refined and the contralateral gland could be spared without hazarding underdosage in the target volume; however, often at the price of still sacrificing the ipsilateral gland [19]. It seems that the paradigm "sacrificing one parotid gland to achieve better sparing of the contralateral gland" often applied with advanced $\mathrm{HNC}$ can be revisited with the advent of VMAT.

From Table 4 we can see that some institutes have only a few dosimetric parameters with significant statistical difference, whereas others have the majority of parameters showing significant differences between VMAT and IMRT. A reason for this may be that only limited efforts were made to homogenize the IMRT techniques among institutes before starting the study. The dosimetric gain of VMAT compared to each of the individual IMRT techniques may therefore differ depending on how complex/ well-designed an IMRT technique of a specific institute was compared to those of other institutes. Keeping these "heterogeneous" sources in mind, the conclusions of the presented study with VMAT leading to better dosimetric results are even more striking.

Limitations of the here presented study include the small sample size in the stratified comparisons, heterogeneity in TPS and equipment of the participating institutes, and limitation to a specific IMRT and VMAT implementation combined with specific linear accelerator equipment. For the comparisons stratified by institutes or patients the sample size of only five institutes and five patients is small, resulting in a low statistical power in the stratified comparisons of VMAT and IMRT. However, the pooled data with $n=25$ allows drawing firm conclusions since for each pairing we can assume statistical independency as different treatment planners are involved. Regarding the heterogeneity of equipment, the two different TPSs employed in this study may yield slightly different results for both IMRT and VMAT plans, although the optimization modules in both TPSs are of the same evolutionary origin. Regarding the different MLCs used for treatment planning, the newer generation beam modulator with narrower leaves of $4 \mathrm{~mm}$ width also allowing interdigitation may lead to better dosimetric results for both IMRT and VMAT, especially regarding dose conformity, due to increased degrees of freedom in leave motion. However, any differences in boundary conditions will be balanced since only pairs of IMRT and VMAT plans achieved under the same conditions are compared. The superior results for VMAT obtained in this study may be valid only for the specific combination of step-and-shoot IMRT and SmartArc/Oncentra VMAT delivered on Elekta linear accelerators equipped with the specified MLCs, and a similar comparison with sliding-window IMRT could lead to different results.

The data presented in this planning study comparing VMAT and static-beam IMRT for HNC resulted from a collaboration of five institutes in the Netherlands with similar equipment, aiming at a safe and fast clinical introduction of VMAT for HNC. Discussion of the planning results and exchange of ideas and information regarding VMAT treatment planning parameters and objectives between participating institutes during the collaboration resulted in noticeable improvement of the VMAT plans. We would like to stress that collaboration between institutes with similar equipment and treatment planning software on the clinical introduction of a new treatment modality can help to efficiently steepen the learning curve and to achieve a high quality of treatment planning within a short time. Problems and questions arising can be solved during the discussions within the group, and as a result all institutes require less time and effort for the clinical introduction of the advanced treatment modality VMAT for HNC.

\section{Conclusions}

VMAT plans including double arcs for simultaneousintegrated boost treatments of head-and-neck cancer were found to be improved compared to static-beam step-andshoot IMRT plans including 5-9 beam ports regarding dose to OARs and dose conformity, while delivery times were significantly shortened by $50 \%$. 


\section{Competing interests}

The authors declare that they have no competing interests.

\section{Authors' contributions}

$\mathrm{AH}$ participated in the study design and study coordination, performed treatment planning, collected and analyzed data, performed the statistical analyses for the study, interpreted data, revised literature and drafted the manuscript. DvG participated in the study design, prepared patient data sets for treatment planning, interpreted data, revised literature and helped draft the manuscript. MA, EK, DS, MK and RL participated in the study design, performed treatment planning, interpreted data, revised literature and helped draft the manuscript. CVV participated in the study design and study coordination, interpreted data, revised literature and helped draft the manuscript. All authors read and approved the final manuscript.

\section{Acknowledgements}

The authors acknowledge Emmy Lamers-Kuijper (The Netherlands Cancer Institute) and Peter van Kollenburg (University Medical Center Nijmegen) for help with treatment planning.

\section{Author details}

'Department of Radiation Oncology, The Netherlands Cancer Institute Antoni van Leeuwenhoek Hospital, Plesmanlaan 121, Amsterdam, CX 1066, The Netherlands. ${ }^{2}$ Department of Radiation Oncology, Antwerp University Radiotherapy, UZA/ZNA, Antwerp, Belgium. ${ }^{3}$ Radiotherapeutic Institute Friesland, Leeuwarden, The Netherlands. ${ }^{4}$ Department of Radiation Oncology, University Medical Center Groningen, University of Groningen, Groningen, The Netherlands. ${ }^{5}$ Department of Radiation Oncology, Catharina Hospital, Eindhoven, The Netherlands. ${ }^{6}$ Department of Radiation Oncology, Radboud University Medical Center Nijmegen, Nijmegen, The Netherlands. ${ }^{7}$ Present address: Department of Radiation Oncology, University of Duisburg-Essen, Essen, Germany. ${ }^{8}$ Present address: Department of Radiation Oncology, Wellington Blood and Cancer Centre, Wellington, New Zealand.

Received: 25 July 2012 Accepted: 28 December 2012

Published: 31 January 2013

\section{Reference}

1. Vergeer MR, Doornaert PA, Rietveld DH, Leemans CR, Slotman BJ, Langendijk JA: Intensity-modulated radiotherapy reduces radiationinduced morbidity and improves health-related quality of life: results of a nonrandomized prospective study using a standardized follow-up program. Int J Radiat Oncol Biol Phys 2009, 74:1-8.

2. van Rij CM, Oughlane-Heemsbergen WD, Ackerstaff AH, Lamers EA, Balm AJ, Rasch CR: Parotid gland sparing IMRT for head and neck cancer improves xerostomia related quality of life. Radiat Oncol 2008, 3:41.

3. Nutting C, A'Hern R, Rogers MS, Sydenham MA, Adab F, Harrington K, Jefferies S, Scrase C, Yap BK, Hall E, PARSPORT Trial Management Group: First results of a phase III multicenter randomized controlled trial of intensity modulated (IMRT) versus conventional radiotherapy (RT) in head and neck cancer (PARSPORT: ISRCTN48243537; CRUK/03/005). J Clin Oncol (Meeting Abstracts) 2009, 27(18S):LBA6006.

4. Braam PM, Terhaard CH, Roesink JM, Raaijmakers CP: Intensity-modulated radiotherapy significantly reduces xerostomia compared with conventional radiotherapy. Int J Radiat Oncol Biol Phys 2006, 66:975-980.

5. Chao KS, Majhail N, Huang CJ, Simpson JR, Perez CA, Haughey B, Spector G: Intensity-modulated radiation therapy reduces late salivary toxicity without compromising tumor control in patients with oropharyngeal carcinoma: a comparison with conventional techniques. Radiother Oncol 2001, 61:275-280.

6. Dijkema T, Terhaard CH, Roesink JM, Braam PM, van Gils CH, Moerland MA, Raaijmakers CP: Large cohort dose-volume response analysis of parotid gland function after radiotherapy: intensity-modulated versus conventional radiotherapy. Int J Radiat Oncol Biol Phys 2008, 72:1101-1109.

7. Braam PM, Roesink JM, Moerland MA, Raaijmakers CP, Schipper M, Terhaard $\mathrm{CH}$ : Long-term parotid gland function after radiotherapy. Int J Radiat Oncol Biol Phys 2005, 62:659-664.

8. Bedford JL: Treatment planning for volumetric modulated arc therapy. Med Phys 2009, 36:5128-5138.

9. Verbakel WF, Cuijpers JP, Hoffmans D, Bieker M, Slotman BJ, Senan S: Volumetric intensity-modulated arc therapy vs. conventional IMRT in head-and-neck cancer: a comparative planning and dosimetric study. Int J Radiat Oncol Biol Phys 2009, 74:252-259.

10. Vanetti E, Clivio A, Nicolini G, Fogliata A, Ghosh-Laskar S, Agarwal JP, Upret RR, Budrukkar A, Murthy V, Deshpande DD, Shrivastava SK, Dinshaw KA, Cozzi L: Volumetric modulated arc radiotherapy for carcinomas of the oro-pharynx, hypo-pharynx and larynx: a treatment planning comparison with fixed field IMRT. Radiother Oncol 2009, 92:111-117.

11. Bertelsen A, Hansen CR, Johansen J, Brink C: Single Arc volumetric modulated Arc therapy of head and neck cancer. Radiother Oncol 2010, 95:142-148.

12. Rao M, Yang W, Chen F, Sheng K, Ye J, Mehta V, Shepard D, Cao D: Comparison of Elekta VMAT with helical tomotherapy and fixed field IMRT: plan quality, delivery efficiency and accuracy. Med Phys 2010, 37:1350-1359.

13. Clemente S, Wu B, Sanguineti G, Fusco V, Ricchetti F, Wong J, McNutt T: SmartArc-based volumetric modulated arc therapy for oropharyngeal cancer: a dosimetric comparison with both intensity-modulated radiation therapy and helical tomotherapy. Int J Radiat Oncol Biol Phys 2011, 80:1248-1255.

14. Lee TF, Chao PJ, Ting HM, Lo SH, Wang YW, Tuan CC, Fang FM, Su TJ: Comparative analysis of SmartArc-based dual arc volumetric-modulated arc radiotherapy (VMAT) versus intensity-modulated radiotherapy (IMRT) for nasopharyngeal carcinoma. J App/ Clin Med Phys 2011, 12:3587.

15. Lu SH, Cheng JC, Kuo SH, Lee JJ, Chen LH, Wu JK, Chen YH, Chen WY, Wen SY, Chong FC, Wu CJ, Wang CW: Volumetric modulated arc therapy for nasopharyngeal carcinoma: A dosimetric comparison with TomoTherapy and step-and-shoot IMRT. Radiother Oncol 2012, 104:324-330.

16. Wiezorek T, Brachwitz T, Georg D, Blank E, Fotina I, Habl G, Kretschmer M, Lutters G, Salz H, Schubert K, Wagner D, Wendt TG: Rotational IMRT techniques compared to fixed gantry IMRT and tomotherapy: multiinstitutional planning study for head-and-neck cases. Radiat Oncol 2011, 6:20.

17. Hoogeman MS, Nuyttens JJ, Levendag PC, Heijmen BJ: Time dependence of intrafraction patient motion assessed by repeat stereoscopic imaging. Int J Radiat Oncol Biol Phys 2008, 70:609-618.

18. Eisbruch A, Ship JA, Martel MK, Ten Haken RK, Marsh LH, Wolf GT, Esclamado RM, Bradford CR, Terrell JE, Gebarski SS, Lichter AS: Parotid gland sparing in patients undergoing bilateral head and neck irradiation: techniques and early results. Int J Radiat Oncol Biol Phys 1996, 36:469-480.

19. Anand AK, Jain J, Negi PS, Chaudhoory AR, Sinha SN, Choudhury PS, Kumar $R$, Munjal RK: Can dose reduction to one parotid gland prevent xerostomia?-a feasibility study for locally advanced head and neck cancer patients treated with intensity-modulated radiotherapy. Clin Oncol (R Coll Radiol) 2006, 18:497-504.

\section{doi:10.1186/1748-717X-8-26}

Cite this article as: Holt et al:: Multi-institutional comparison of volumetric modulated arc therapy vs. intensity-modulated radiation therapy for head-and-neck cancer: a planning study. Radiation Oncology 2013 8:26.

\section{Submit your next manuscript to BioMed Central and take full advantage of:}

- Convenient online submission

- Thorough peer review

- No space constraints or color figure charges

- Immediate publication on acceptance

- Inclusion in PubMed, CAS, Scopus and Google Scholar

- Research which is freely available for redistribution 